\title{
Probing the 5th Dimension with the QCD String *
}

\author{
Richard C. Brower \\ Physics Department \\ Chung-I Tan \\ Boston University \\ Boston, MA 02215, USA \\ Physics Department \\ Brown University \\ Providence, RI 02912, USA \\ Ethan Thompson \\ Department of Physics \\ University of Washington \\ Seattle, WA 98195, USA
}

October 2, 2018

\begin{abstract}
A salient feature of String/Gauge duality is an extra 5th dimension. Here we study the effect of confining deformations of $A d S^{5}$ and compute the spectrum of a string stretched between infinitely massive quarks and compare it with the quantum states of the QCD flux as determined by Kuti, Juge and Morningstar in lattice simulations. In the long flux tube limit the AdS string probes the metric near the IR cutoff of the 5th dimension with a spectrum approximated by a Nambu-Goto string in 4-d flat space, whereas at short distance the string moves to the UV region with a discrete spectrum for pure $A d S^{5}$. We also review earlier results on glueballs states and the cross-over between hard and soft diffractive scattering that support this picture.
\end{abstract}

\footnotetext{
*Brown-HET-1431: This work was supported in part by the Department of Energy under Contracts No. DE-FG02-91ER40676 and No. DE-FG02-91ER40688
} 


\section{Introduction}

The picture for QCD based on String/Gauge duality (see below for some technical details) proceeds as follows. The flux tube of QCD is dual to a fundamental string (or world sheet sigma model) in 5 dimensional AdS like space plus 5 compact dimensions for the critical superstring. The motion of the string in 4-d space time $x^{\mu}$ is accompanied by motion in a transverse "radial" co-ordinate, $r$. The inverse radial co-ordinate $z=R^{2} / r$ (scaled by the AdS radius $R$ ) represents conformal transformations in the Yang-Mills theory. UV physics is mapped into small z (large $\mathrm{r}$ ) and IR physics into large $\mathrm{z}$ (small $\mathrm{r}$ ). The minimal energy of the stretched string is determined by the geodesic in the $\mathrm{x}-\mathrm{z}$ space. Its quantum fluctuations give the spectrum for the flux tube of Yang Mills theory.

Remarkably we find that these transverse fluctuations obey a simple wave equation,

$$
\left[\partial_{t}^{2}-v^{2}(\sigma) \partial_{\sigma}^{2}\right] X^{\perp}=0
$$

where $\sigma$ is the proper distance along the flux tube as seen by the Yang-Mills observer. Independent of the precise warping of the space in the 5 th co-ordinate, the local velocity takes a universal form,

$$
v(\sigma)=\frac{z^{2}(\sigma)}{z_{c}^{2}} .
$$

It is given by the square of the displacement in $z(\sigma)$ of the minimal surface in the $x-z$ plane. For a long flux tube relative to the confinement scale, the velocity is nearly constant and equal to the speed of light $\left(z \simeq z_{c}\right)$ so the physics is well approximated by a naive massless (Nambu-Goto) string in 4-d with energy levels $\Delta E_{n} \simeq \pi(n-1 / 12) / L+O\left(1 / L^{3}\right)$. However, the signal slows as it enters the environment of the massive quark. For small $Q-\bar{Q}$ separation, the classical energy is Coulombic and the fluctuations are controlled by the CFT limit.

The goal of this talk is to show how this picture transpires.

\section{String/Gauge Duality}

In 1998 Maldacena 1, 2, 3] took a decisive step toward realizing the "ancient" quest for the QCD string. Under specific circumstances he gave strong arguments for the "String/Gauge" conjecture of an exact equivalence (aka duality) between 10-d superstring theory in a curved background and super Yang-Mills theory in 4-d. Subsequent computations in the last few years 
have given hundreds of specific tests of this conjecture and a myriad of extensions to a large class of Yang-Mills theories. This cast the question of the existence of the QCD string in an entirely new light. We now have a qualitative road map for how such an equivalence may be realized without to date a precise construction of the string dual to QCD. It is interesting to explore the physical consequences of this qualitative picture for $\mathrm{QCD}$, trying to extract features that are independent of the detailed realization not yet available for analysis.

Maldacena's original celebrated example of the duality between IIB string theory in an $A d S^{5} \times S^{5}$ background metric,

$$
d s^{2}=\frac{r^{2}}{R^{2}} \eta_{\mu \nu} d X^{\mu} d X^{\nu}+\frac{R^{2}}{r^{2}}\left(d r^{2}+r^{2} d^{2} \Omega_{5}\right)
$$

and $\mathcal{N}=4$ Super Yang-Mills still provides a useful prototype. The essential feature is the addition of a "5th radial" dimension, $r$, transverse to the $3+1$ Minkowski space, $X^{\mu}$. Large $\mathrm{r}$ is UV physics for the dual Yang-Mills, while small $\mathrm{r}$ is the IR region. In $A d S^{5} \times S^{5}$ the isometries of the background, $S O(3,2) \times S O(6)$, are dual to the conformal group times the R symmetries of $\mathcal{N}=4$ SUSY Yang-Mills. The 'tHooft coupling,

$$
\lambda \equiv g^{2} N_{c}=4 \pi g_{s} N=R^{4} / l_{s}^{4},
$$

in Yang-Mills theory is expressed in terms of the closed string coupling $g_{s}$, the AdS radius $R$ and the string length $l_{s}$. (The open string tension is $T_{s}=\left(2 \pi \alpha_{s}^{\prime}\right)^{-1}$ in terms of the Regge slope, $\alpha_{s}^{\prime}=l_{s}^{2}$. Another common convention defines $g_{Y M}^{2}=2 \pi g_{s}$.) This highly symmetric case is amenable to many analytic tools which have greatly elucidated String/Gauge duality. However pure $S U\left(N_{c}\right)$ Yang-Mills for quarkless QCD has much less symmetry. There is no super symmetry and conformal symmetry is broken by quantum effects giving rise to "dimensional transmutation", asymptotic freedom and confinement. Backgrounds that break conformal and some or all SUSY symmetries differ in the precise details of the "warping" of the 5th dimension.

Yang-Mills operators correspond to boundary conditions on the string (or super gravity) Green's function at $r \rightarrow \infty$. It is sometimes more convenient to invert the radial co-ordinate by introducing $z=R^{2} / r$, placing this boundary at $z=0$,

$$
d s^{2}=\frac{R^{2}}{z^{2}}\left(\eta_{\mu \nu} d X^{\mu} d X^{\nu}+d z^{2}\right)+R^{2} d^{2} \Omega_{5} .
$$

Surprisingly, the conformal example exhibits stringy physics in spite of its lack of confinement (and the stringy idea of narrow electric flux tubes in the Yang-Mills description). Due to conformal symmetry the potential between two heavy "quark" sources at separation L is purely Coulombic,

$$
E_{0}(L)=-\frac{(2 \pi)^{2}}{\Gamma^{4}(1 / 4)} \frac{\sqrt{2 g^{2} N_{c}}}{L}
$$


That is, the Wilson loop has perimeter rather than area law behavior even as L goes to infinity. At short distances this potential is not a bad description of QCD up to the asymptotically free logarithmic corrections and there is a discrete set of excited modes above the ground states as described in Sec. 4 .

A useful prototype for a class of confining deformations can be expressed as

$$
d s^{2}=\frac{R^{2}}{z^{2}}\left[\eta_{\mu \nu} d X^{\mu} d X^{\nu}+G^{2}\left(z / z_{\max }\right) d z^{2}\right]+\text { compact dimension }
$$

which approaches $A d S^{5}$ in the $\mathrm{UV},\left(G^{2}\left(z / z_{\max } \rightarrow 0\right)=1\right)$, but is strongly modified in the IR with a cutoff at $r_{\min }=R^{2} / z_{\max }$. The new mass scale, $\Lambda_{I R} \equiv r_{\min } / R^{2}$, is proportional to the mass gap or lowest glueball mass in the spectrum and plays the role of $\Lambda_{q c d}$. The simplest phenomenological model, exploited extensively in the work of Polchinski and Strassler 4, uses the pure $A d S^{5}$ metric for $r \geq r_{\min }$ with a "square-well" cutoff at $r=r_{\min }$ (Dirichlet condition on super gravity fields),

$$
G_{\text {cutoff }}\left(z / z_{\max }\right)=\theta\left(1-z / z_{\max }\right) .
$$

Here we use a softer IR cutoff inspired by an $A d S^{\gamma+1}$ Euclidean black hole confining background metric,

$$
G_{\text {blackhole }}\left(z / z_{\max }\right)=\frac{1}{\sqrt{1-\left(z / z_{\max }\right)^{\gamma}}} .
$$

A major consequence of this IR cutoff besides obviously introducing a scale that breaks conformal symmetry is confinement.

\section{Qualitative Results for Confining Backgrounds}

The early calculations in a confining background included the glueball spectrum [5, 6, 7, 8] (at strong coupling) and a heuristic picture of wide angle scattering [9, 10]. By comparing these calculations with lattice spectra for glueballs on the one hand and experimental data for hard scattering on the other (interpreted by parton models at weak coupling), one can begin to understand the qualitative physics of QCD like string models in deformed AdS space and their

limitations. For instance, the glueball wave functions are concentrated near $r=r_{\text {min }}$, reflecting IR physics, whereas hard scattering $(s \sim-t)$ is dominated by $r^{2} \geq r_{\text {scatt }}^{2} \sim \alpha_{s}^{\prime} R^{2} s$. Together they probe two opposite limits of the 5 th dimension [11]. 


\subsection{Glueball Spectra}

The computation of the glueball spectrum in strong coupling for the $A d S^{7}$ black hole (i.e., Eq. 2 with $\gamma=6$, [8] ) provided the first detailed application of String/Gauge duality for confining theories. From the effective Born-Infeld action on the brane,

$$
S=\int d^{5} x \operatorname{det}\left[G_{\mu \nu}+e^{-\phi / 2}\left(B_{\mu \nu}+F_{\mu \nu}\right)\right]+\int d^{4} x\left(C_{1} F \wedge F+C_{3} \wedge F+C_{5}\right),
$$

one can read off the quantum numbers in the dual Yang-Mills theory. The entire spectrum for all states in the QCD super selection sector were then compared with lattice data [12] for SU(3). In spite of the crude approximations of strong coupling, the results are rather encouraging (see Fig. 11). All the states are in the correct relative order and the missing states at higher J are a direct consequence of strong coupling which pushes the string tension to infinity. It appears
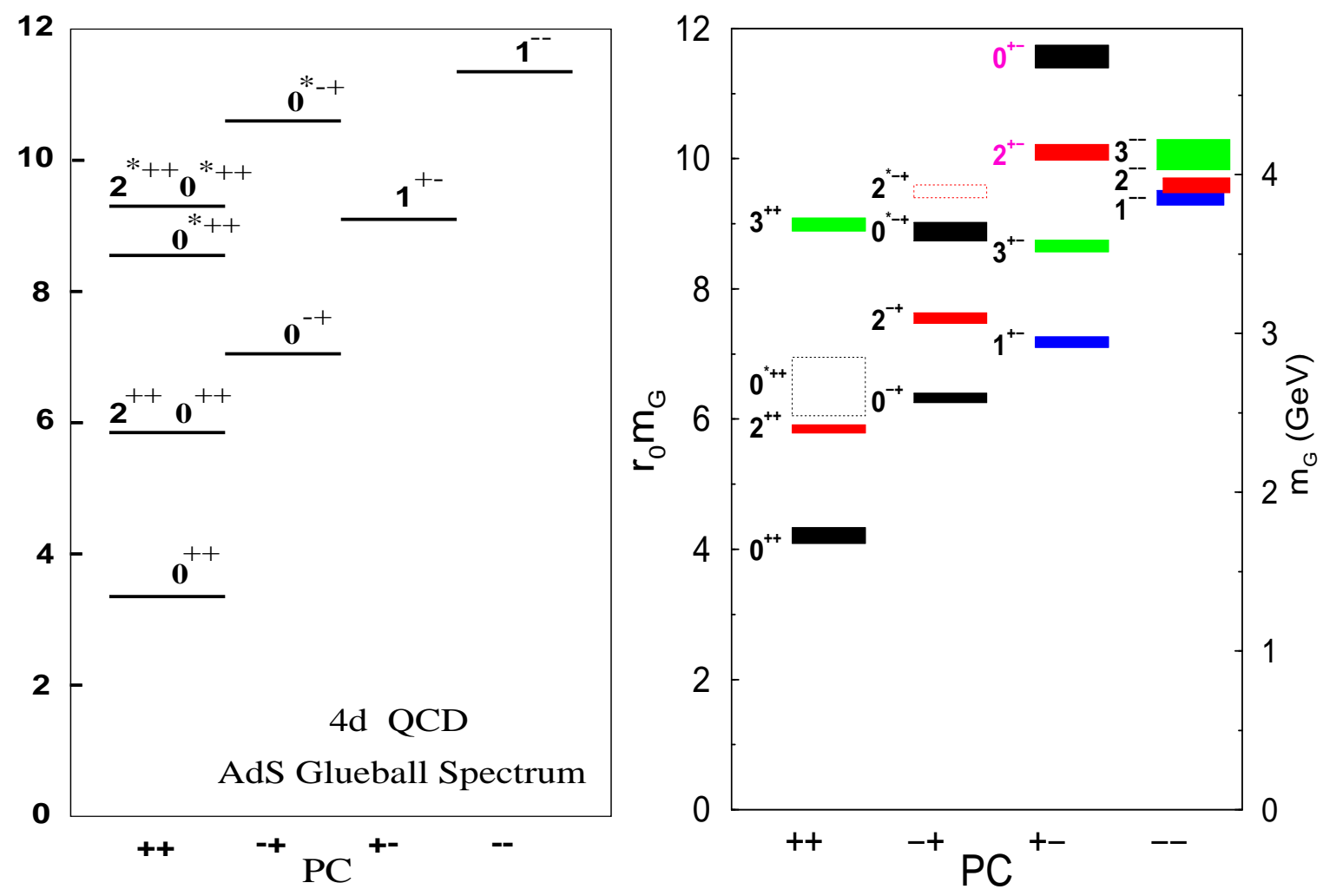

Figure 1: The AdS glueball spectrum for $Q C D_{4}$ in strong coupling (left) compared with the lattice spectrum [12] for pure SU(3) QCD (right). The AdS cutoff scale is adjusted to set the lowest $2^{++}$tensor state to the lattice results in units of the hadronic scale $1 / r_{0}=410 \mathrm{Mev}$.

plausible that the $A d S^{7}$ black hole phase at strong coupling is rather smoothly connected to the weak coupling (confined) fixed point of QCD. However it must be emphasized that there is no general understanding of how the metric can be deformed so that all the unwanted charged 
Kaluza-Klein states in the extra compact directions decouple. So far all attempts to find better background solutions to supergravity as a starting point for QCD have failed in this regard.

\subsection{Near-Forward Scattering and Regge Behavior}

An important paper by Polchinski and Strassler [9] began to explain how hard scattering can be accommodated in the string description in spite of the exponentially soft behavior for all string scattering amplitudes in flat space. By assuming a single local scattering in a cutoff $A d S^{5}$ background, the glueball elastic scattering amplitude, $T(s, t)$, takes the form $T(s, t)=$ $\int_{r_{h}}^{\infty} d r \mathcal{K}(r) A(\hat{s}, \hat{t}, r)$, where $A$ is a local 4-point amplitude for string scattering at $r$ and $\mathcal{K}(r)$ accounts for the phase space and glueball wave functions. In the local frame for the string scattering amplitude, the Mandelstam invariants are $\hat{s}=(R / r)^{2} s$ and $\hat{t}=(R / r)^{2} t$, due to the red-shift in $\mathrm{r}$.

In the Regge limit the amplitude becomes

$$
T(s, t)=\int_{r_{h}}^{\infty} d r \mathcal{K}(r) \beta(\hat{t})\left(\alpha_{s}^{\prime} \hat{s}\right)^{\alpha_{0}+\frac{1}{2} \alpha_{s}^{\prime} \hat{t}}
$$

As further explained by Brower and Tan [10, for small $t \simeq 0$, this corresponds to an exchange of a BFKL-like Pomeron, with a small effective Regge slope,

$$
\alpha_{B F K L}^{\prime}(0) \sim \frac{1}{2}\left(r_{m i n} / r_{h}\right)^{2} \alpha_{q c d}^{\prime}<<\alpha_{q c d}^{\prime}
$$

Such an exchange naturally leads to an elastic diffraction peak with little shrinkage [13. In the coordinate space, one finds, for a hard process, the transverse size is given by

$$
<\vec{X}^{2}>\sim \frac{1}{2}\left(r_{\min } / r_{h}\right)^{2} \alpha_{q c d}^{\prime} \log s+\text { constant } .
$$

If the cutoff, $r_{h}$, which characterizes a hard process, increases mildly with $s$ ( e.g. $r_{h}^{2} \sim \log s$, there will be no transverse spread.

They also note that glueball wave functions are large in the IR so that there is a strong scattering amplitude for $r \simeq r_{\min }$,

$$
T(s, t) \sim A\left(s, t, r_{\min }\right) \sim\left(\alpha_{q c d}^{\prime} s\right)^{\alpha_{P}(0)+\frac{1}{2} \alpha_{q c d}^{\prime} t},
$$

approximating the soft Regge pole [10]. The QCD Regge slope to first non-trivial order in strong coupling is

$$
\alpha_{q c d}^{\prime}=\frac{R^{2}}{r_{\min }^{2}} \alpha_{s}^{\prime}=\frac{1}{\Lambda_{I R}^{2}} \frac{\alpha_{s}^{\prime}}{R^{2}}=\frac{1}{\Lambda_{I R}^{2} \sqrt{g^{2} N_{c}}}
$$


consistent with the surface tension of the open string, $T_{q c d}=\left(2 \pi \alpha_{q c d}^{\prime}\right)^{-1}$, computed by the area law for a Wilson loop [1, 14, 15]. The linear extrapolation from the glueball gives an estimate for the intercept of

$$
\alpha_{P}(0)=2-\frac{1}{2} \alpha_{q c d}^{\prime} M_{G B}^{2}=2-\frac{2 M_{G B}^{2}}{\Lambda_{I R}^{2} \sqrt{g^{2} N_{c}}},
$$

where $M_{G B}^{2}$ is the mass of the tensor $2^{++}$glueball. Of course, the true Regge pole, that gives the zero-width physical states at $N_{c}=\infty$ string theory, must give a pure Regge power at fixed $t$ with exact factorization in the t-channel [13] in contradiction with the approximate amplitude in Eq. 10] above. This cannot be achieved in the local string scattering approximation at fixed r. Departures from this local approximation are also necessary to really probe partonic features in deep inelastic scattering [4, since constituent pieces of the string need to be isolated in the UV region. The consequences for diffractive scattering and the BFKL Pomeron of going beyond the local approximation is under active investigation [11].

\section{Stretched String Spectra}

An even more direct probe of the 5 -th dimension is provided by the spectrum of the string stretched between infinitely heavy sources (see Fig 2). From the AdS/CFT viewpoint, starting with the ends of the string separated by a small distance L, we are probing the short distance Coulomb regime. Then as we increase L, the minimal surface moves into the interior probing more and more IR physics. Finally at very large L we see only the lowest mass transverse "Goldstone modes" of the string leading to the universal spectrum of Lüscher,

$$
E_{n}=T_{0} L+\frac{\pi}{L}\left[a_{n}^{\perp \dagger} a_{n}^{\perp}-\frac{(D-2)}{24}\right]+\cdots
$$

Indeed at large separation $\mathrm{L}$ the lattice data for the stretched string spectrum appears to be approaching this form with 2 transverse oscillators, D-2 = 2. (See Fig. 2). Using a very cleaver lattice simulation method, Lüscher and Weisz [17] were apparently able to determine the one loop contribution ( i.e.the so called "Lüscher term") to the ground state,

$$
E_{0}^{1 l o o p}(L)=-\frac{\pi}{12}(1+0.12 f m / L)
$$

for $\mathrm{L}$ in the range 0.5 to $1.0 \mathrm{fm}$. The agreement with theory for $D-2=2$ is remarkable (if not paradoxical) in view of large non-universal features in the spectrum for $L \simeq 0.5-1.0 \mathrm{fm}$ clearly visible in the lattice data (Fig (2).

It is evident from the lattice data in Fig. 2 that a major challenge for the AdS/CFT approach to the QCD string is to understand the highly non-trivial interpolation between IR 


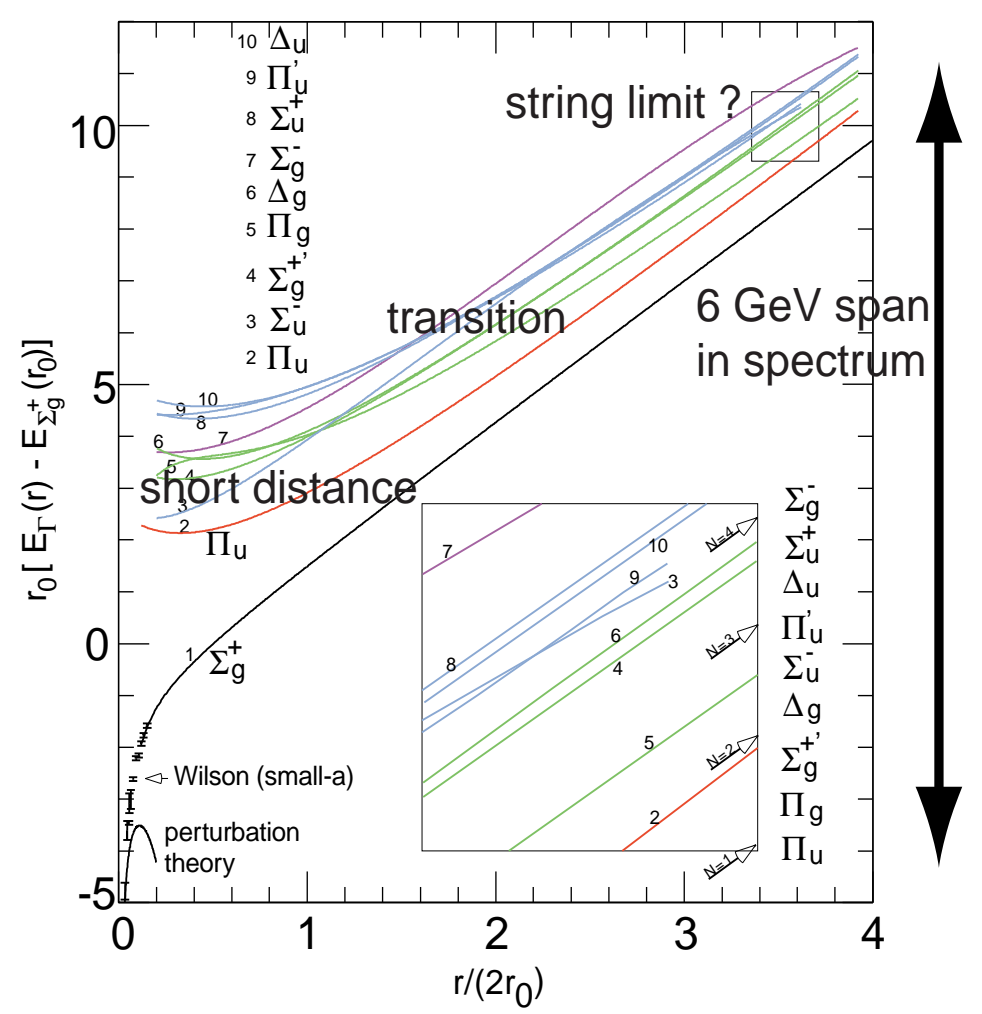

Figure 2: Lattice string spectrum for SU(3) Yang-Mills theory determined by Juge, Kuti and Moringstar [16]

(large L) and UV (small L) physics of the string. As a first attempt, we are quantizing the string with Dirichlet boundary conditions in the confining $A d S^{5}$ blackhole metric of Eq. [6] above. First we solve (numerically) for the minimal surface getting a classical potential energy, $E_{0}^{\text {class }}(L)$ for the ground state of the stretched string. This function obeys the following limits,

$$
E_{0}^{\text {class }}(L \rightarrow \infty) \simeq \frac{r_{\min }^{2}}{2 \pi \alpha_{s}^{\prime} R^{2}} L+O\left(L e^{-c L}\right) \quad \text { and } \quad E_{0}^{\text {class }}(L \rightarrow 0) \simeq-\frac{\pi^{2} \sqrt{2}}{\alpha_{s}^{\prime} \Gamma(1 / 4)^{4}} \frac{R^{2}}{L}
$$

The exact function, $E_{0}^{\text {class }}(L)$, fits almost perfectly the lattice data for all $\mathrm{L}$, after adjusting the mass scale $R^{2} / r_{\text {min }}=\Lambda_{I R}$ and the Regge slope $\alpha_{q c d}^{\prime}=R^{2} \alpha_{s}^{\prime} / r_{m i n}^{2}$. This is reassuring but also highlights the present weakness of our AdS model. Pure Yang-Mills (quarkless QCD) predicts a definite number for the string tension, $T_{q c d}=1 /\left(2 \pi \alpha_{q c d}^{\prime}\right)$ relative to the single Yang-Mills scale, $\Lambda_{q c d}$. However at present all AdS/CFT strong coupling models for confining theory have an extra cutoff parameter that can be adjusted independent of the Regge slope (or QCD string tension). This extra mass scale is similar to strong coupling lattice QCD, except that on the lattice the new scale is the UV cutoff of the lattice spacing whereas here it is a phenomenological IR scale more similar to the MIT bag constant.

Work is currently underway to compute the energy levels, $E_{n}=E_{0}^{\text {class }}+\Delta E_{n}$, above 
the ground state in our model AdS blackhole background. In a temporal gauge $X^{(0)}=t$ with $\sigma=X^{(3)}$, the linearized or semi-classical equation for the transverse fluctuations of a string stretched is

$$
\left[\partial_{t}^{2}-v^{2}(\sigma) \partial_{\sigma}^{2}\right] X^{\perp}(t, \sigma)=0
$$

The local velocity, $v(\sigma)=z^{2}(\sigma) / z_{c}^{2}$, of propagation along the string is bounded by the speed of light. At large L, the velocity approaches a constant, exponentially close to the speed of light, except near the quarks at $z=0$. Thus, it is not surprising that at large $\mathrm{L}$ this confining background reproduces flat string results,

$$
\Delta E_{n}=\frac{n \pi}{L}
$$

and in one loop, the universal Lüscher term (or Casimire energy), $\Delta E_{n}^{1 l o o p}=(D-2) \pi / 24$. Moreover a numerical solution to our equation in Fig. 3] shows deviations from the large $\mathrm{L}$ asymptotic value in qualitative agreement with that measured in the Lattice simulations of Juge, Kuti and Morningstar [16]. However there is another contribution to this deviation with

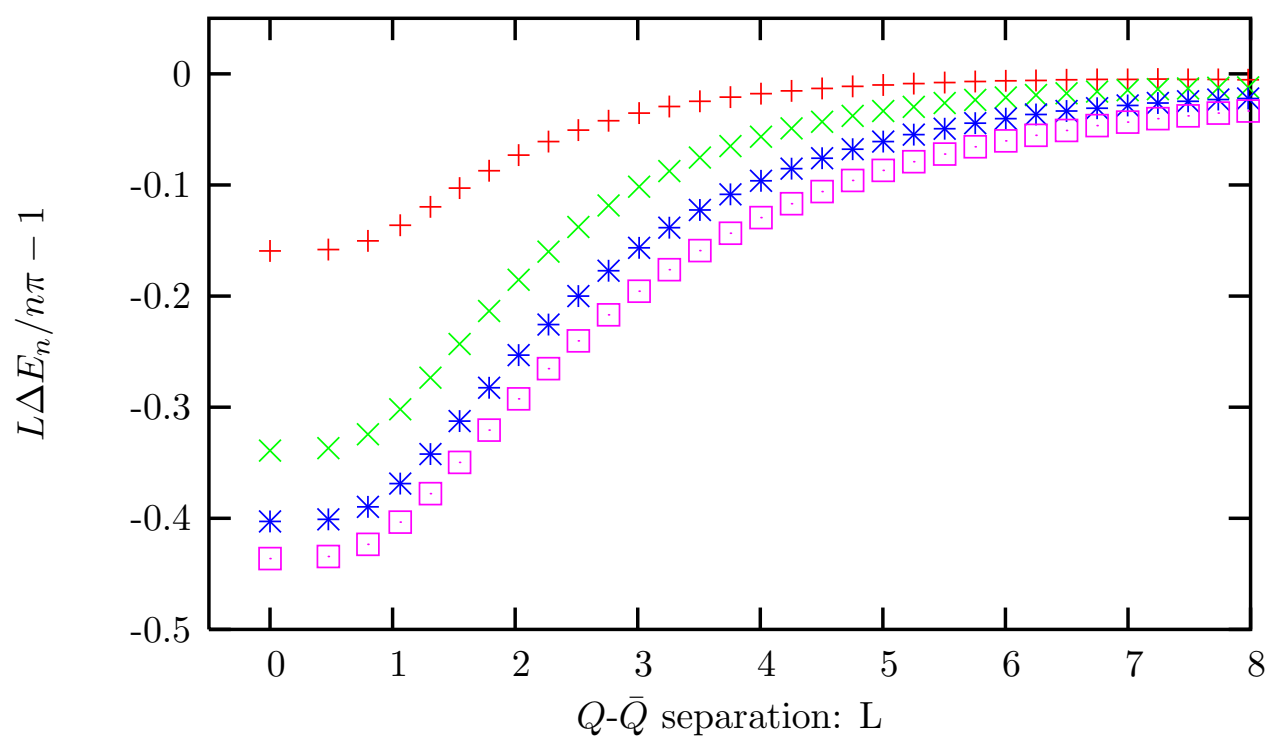

Figure 3: Deviations of transverse energy levels, $L \Delta E_{n} /(n \pi)-1$, in confining $A d S^{5}$ black hole model for $\mathrm{n}=1(+), 2(\times), 3(*)$ and $4(\square)$.

the same sign observed in the exact spectrum of the (flat space) Nambu-Goto

$$
E_{n}^{\text {Nambu-Goto }}=T_{0} L \sqrt{1+\frac{2 \pi}{L}\left[a_{n}^{\perp \dagger} a_{n}^{\perp}-\frac{(D-2)}{24}\right]}
$$

when quantized in the lightcone gauge [19]. An effort is underway to combine these two effects in a self-consistent lightcone quantization of the string in a confining AdS space [18. 
As you approach zero separation $L \rightarrow 0$, the discrete spectrum of the stretched string is determined by the pure $A d S^{5}$ metric [18. The exact spectrum is know in closed form through the sum rule,

$$
z_{c} \Delta E_{n} \sqrt{\left(z_{c} \Delta E_{n}\right)^{4}-1} \int_{0}^{1} \frac{d s}{\left[1+\left(z_{c} \Delta E_{n}\right)^{2}\right] \sqrt{\left(1-s^{2}\right)}}=\frac{n \pi}{2},
$$

give by Callan and Guijosa [20] where $n=1,2, \cdots$ and $z_{c}=(2 \pi)^{-3 / 2} \Gamma\left(\frac{1}{4}\right) L$. In the conformal limit the energies must of course be proportional $1 / L$.

In addition to transverse Goldstone modes, there are quantum modes for fluctuations in the extra "radial" direction,

$$
\left[\partial_{t}^{2}-v^{2}(\sigma) \partial_{\sigma}^{2}\right] \rho(t, \sigma)=M^{2}(\sigma) \rho(t, \sigma)
$$

Unlike the transverse Goldstone modes $X^{\perp}$, now there is a $\sigma$-dependent "rest mass",

$$
M^{2}(\sigma)=\frac{1}{z_{c}^{2}}\left[z \frac{d}{d z} \frac{1}{G^{2}}-\frac{2 z^{4}}{z_{c}^{4} G^{2}}\right]
$$

whose scale is set by the glueball mass: $M_{G B} \sim 1 / z_{c}$. These modes correspond via the String/Gauge duality to longitudinal (breathing) modes for a fat chromodynamic flux tube. It would be interesting to find these modes in lattice gauge theory simulations and trace their dependence on $\mathrm{L}$.

Still our toy QCD string in an $A d S^{5}$ blackhole is at best just a first step in understanding how a QCD string in warped space might behave. Much work remains even to identify the microscopic degrees of freedom of the QCD string let alone to the discovery of an effective string action capable of reproducing the lattice spectrum from long distance into the short distance region governed by asymptotically free gauge theory at large $N_{c}$. However a reasonable near term goal is to find accurate interpolation formulae for all $\mathrm{L}$ for the low energy spectrum of the stretched sting in a legitimate confining super gravity background. On the basis of the comparison of this spectrum with lattice data one might narrow the search for the QCD background geometry itself.

Acknowledgment: We wish to thank Joe Polchinski, Matt Strassler and Charles Thorn for very interesting discussions during our visit to the KITP Workshop on $Q C D$ and String Theory where this draft was written. Also the work on the string spectrum benefited substantially from our interactions with Oliver Jahn and Carlos Nuños at Massachusetts Institute of Technology. 


\section{References}

[1] J. Maldacena, Adv. Theor. Math. Phys. (1998), 231.

[2] E. Witten, Adv. Theor. Math. Phys.2 (1998) 505 ; Adv. Theor. Math. Phys.2 (1998) 253.

[3] S.S. Gubser, I.R. Klebanov and A.M. Polyakov, Phys. Lett. B428 (1998) 105.

[4] Joseph Polchinski and Matthew J. Strassler, "Deep Inelastic Scattering and Gauge/String Duality", JHEP 0305 (2003) 012, hep-th/0209211.

[5] C. Csáki, H. Ooguri, Y. Oz and J. Terning, hep-th/9806021.

[6] R. De Mello Koch, A. Jevicki, M. Mihailescu and J. Nunes, hep-th/9806125

[7] R.C. Brower, S. Mathur and C-I Tan, Nucl. Phys. B 574 (2000) 219, hep-th/9908196.

[8] R.C. Brower, S. Mathur and C-I Tan, "Glueball Spectrum for QCD from AdS Supergravity Duality", Nucl. Phys. B587 (2000) 249, hep-th/0003115.

[9] Joseph Polchinski and Matthew J. Strassler, "Hard Scattering and Gauge/String Duality", Phys.Rev.Lett. 88 (2002) 031601, hep-th/0109174.

[10] R.C. Brower and C-I Tan, "Hard Scattering in the M-Theory Dual for the QCD String", Nucl. Phys., B662 (2003) 393, hep-th/0207144.

[11] Current work in collaboration with J. Polchinski and M. Strassler is revealing a much clearer understanding of how to probe AdS in the Regge scattering limit. Also see Ref. 44 for the use of DIS as a probe.

[12] C. J. Morningstar and M. Peardon, "The glueball spectrum from an anisotropic lattice study", Phys.Rev. D60 (1999) 034509.

[13] C-I Tan, "Diffractive Production at Collider Energies and Factorization", Phys. Report, 315 (1999) 175. For a review on experimental support for identifying the Pomeron Regge trajectory as a closed-string exchange in large-N QCD, see: A. Capella, U. Sukhatme, C-I Tan, and J.T.V. Tran, Phys. Report, 236 (1994) 225.

[14] A. Brandhuber, N. Itzhaki, J. Sonnenschein, and S. Yankielowicz, "Wilson Loops, Confinement, and Phase Transitions in Large N Gauge Theories from Supergravity", JHEP 9806 (1998) 001, hep-th/9803263.

[15] D. Gross and H. Ooguri, Phys. Rev. D58 (1988) 106002, hep-th/9805129, M. Zyskin, Phys. Lett. B439 (1998) 373, hep-th/9806128, H. Ooguri, H. Robins and J. Tannenhauser, Phys. Lett. B437 (1998) 77, hep-th/9806171, J.G. Russo, hep-th/9808117, A. Hashimoto and Y. Oz, hep-th/9809106, C. Csáki, H. Ooguri, Y. Oz and J. Terning, hep-th/9810186, S.S. 
Gubser, hep-th/9810225, P. Kraus, F. Larsen and S.P. Trivedi, hep-th/9811120, R.G. Cai and K.S. Soh, hep-th/9812121, J.G. Russo and K. Sfetsos, hep-th/9901056, C. Csáki, J. Russo, K. Sfetsos and J. Terning, hep-th/9902067.

[16] K. Jimmy Juge, Julius Kuti and Colin Morningstar "The QCD String Spectrum and Conformal Field Theory" Nucl.Phys.Proc.Suppl. 106 (2002) 691-693.

[17] M. Lüscher and P. Weisz "Quark confinement and the bosonic string", JHEP 07 (2002) 049.

[18] Work in progress by R. C. Brower, C-I Tan and C. Thorn on the lightcone string quantiztion in AdS.

[19] J. F. Arvis," The exact q anti-q potential in Nambu String Theory",Phys. Lett.B127 (1983) 106.

[20] Curtis G. Callan and Alberto Guijosa, "Undulating Strings and Gauge Theory Waves", Nucl. Phys. B565 (2000) 157, hep-th/9906153. 